\title{
Des communicants en quête de savoirs et de pratiques réflexives dans une entreprise en tension
}

\author{
Brulois Vincent, \\ LabSIC, Université Paris 13, Sorbonne Paris Cité, \\ France,brulois@sic.univ-paris13.fr \\ Charpentier, Jean-Marie, responsable de \\ l'observation sociale d'une entreprise publique, \\ Vice-président de l'AFCl, France, cojm@club- \\ internet.fr \\ Viers Jacques, \\ Vice-président de l'APSE, France \\ jacques.viers@gmail.com
}




\section{Résumé}

En 2013, l'AFCI organisait la quatrième formation autour de l'apport des sciences sociales à la communication. Celle-ci s'inscrit dans une réflexion engagée auparavant sur les savoirs nécessaires aux pratiques professionnelles des communicants. Son objectif est de leur proposer des éléments de connaissances, en contrepoint de leurs compétences, afin de penser la communication et d'agir dans une période incertaine. Partant des situations de travail des communicants, il s'agit de pousser plus avant la réflexivité sur leurs pratiques, de les aider à comprendre leurs logiques d'action, à se mettre à distance et d'étayer une posture professionnelle différente. À tout le moins, ces deux expériences montrent des acteurs en réflexion et un besoin de quelque chose en contrepoint d'une formation initiale parfois lointaine, souvent pratique et opérationnelle. Elles permettent en tout cas d'apprécier une dynamique de métier à l'œuvre, déportant les enjeux de la communication de plus en plus du côté du dialogue, du débat, voire de la confrontation avec les acteurs de la société comme avec les salariés. S'impose l'idée qu'un détour par les sciences sociales peut favoriser la compréhension de ce monde en mouvement qu'est l'entreprise. In fine, cela peut nourrir d'autres pratiques professionnelles en communication, moins ancrées sur la belle image et le discours lisse que sur le social.

Mots clés : Communication, entreprise, savoir-faire, savoir, réflexivité.

\section{Abstract}

In 2013, the AFCI organized the fourth training around the contribution of the social sciences to communication. This one joins in a reflection committed previously on the knowledge necessary for the professional practices of the communicators. Its objective is to propose them elements of knowledge, in counterpoint of their skills, to think of the communication and act for the uncertain period. Starting work situations of communicators, it is a question of urging more before the reflexivity on their practices, of helping them to understand their logics of action, to get away and to support a different professional posture. At the very least, these two experiments show actors in reflection and need for something in counterpoint of a sometime distant initial formation. They allow anyway estimating dynamics of job in the work, deporting the stakes in the communication on the side of the dialogue, debate or confrontation with the actors of the society as with the employees. The idea is imperative that a bend by the social sciences can favor the understanding of this world in movement that is the company. In fine, it can feed other professional practices in communication, less anchored on the beautiful image and the smooth speech that on the social.

Keywords: company, communication, knowledge, skills, reflexivity 
La position des communicants internes évolue aujourd'hui dans une entreprise en tension. Leur situation souvent singulière, difficile parfois, interroge à la fois la réalité de leur métier et ce dont ont besoin ces professionnels pour l'exercer. Pardelà les compétences, les savoir-faire professionnels classiquement requis ou sollicités, des communicants témoignent d'un intérêt nouveau pour des savoirs, des connaissances en sciences humaines et sociales (SHS) ainsi que pour des démarches réflexives de type analyse de pratiques.

L'entreprise est en tension car le changement y est continu. Afin de s'adapter à des complexités financières, organisationnelles, sociales et territoriales, elle se transforme en permanence. Elle adopte de nouvelles stratégies qui entraînent de nouvelles organisations, celles-ci se succédant à un rythme très rapide. Toutes ces transformations, en forme de «mouvement permanent qui mobilise l'ensemble des acteurs » (Alter, 2000), posent de redoutables questions de sens, que ce soit en termes de direction, de signification ou d'identité pour elle-même comme pour les salariés. Par ailleurs, sous l'égide de la corporate governance, la notion même d'entreprise s'est transformée, banalisant l'idée que son objectif n'est ni plus ni moins que de «faire du profit» (Segrestin et Hatchuel, 2012) et de servir d'abord l'intérêt de l'actionnaire. Selon ces auteurs, la crise financière de ces dernières années apparaît ainsi comme résultant en grande part d'une «crise de l'entreprise et de sa gestion ». Les salariés ne manquent pas de faire le lien entre ce qu'ils vivent dans l'entreprise et ce qu'ils perçoivent dans la société. Ils sont à plusieurs titres «concernés» (Callon et alii, 2001), laissant moins qu'avant au vestiaire de l'entreprise les habits qu'ils portent dans la société : citoyen, militant associatif, père ou mère de famille, riverain. Et tout ce qui pouvait hier être abordé, non sans mal, par le monologue descendant appelle désormais discussion et dialogue. Ils n'acceptent plus le changement perpétuel sans mot dire, ne s'en laissent plus conter, se méfient, interrogent, cherchent à "prendre l'entreprise au piège de ses communications antérieures »(Basilien et alii, 2010), n'hésitant plus à mettre en défiance la moindre action, la moindre parole de leur entreprise.

Dans ce champ de tensions, le communicant est aux premières loges. Il a à gérer un écart entre le politiquement correct du discours économique et financier de l'entreprise, auquel il est tenu, et des enjeux sociaux de plus en plus prégnants. Comme d'autres, il constate que l'entreprise est aux prises avec des complexités de plus en plus fortes. Mais, plus que d'autres, il vérifie au quotidien la difficulté de communiquer ces complexités quand sa hiérarchie attend de lui une communication monologique, instrumentale, fortement simplificatrice. Quand on parle communication en entreprise, il n'est souvent question que de messages à transmettre, de cibles à identifier, d'éléments de langage à utiliser, de marque à rendre visible, mais aussi d'outils, de supports ou de dispositifs à choisir en fonction des messages et des cibles. Vision éminemment réductrice où la communication se confond avec un processus de création et de circulation de messages vers des 
destinataires qu'il s'agit d'informer, de convaincre, d'influencer, voire de manipuler - faire adhérer aux changements -, mais bien peu d'écouter. Ce processus a plus à voir avec la transmission qu'avec la communication. Malgré les changements d'époques, voire de technologies l'idée persiste qu'établir une stratégie de communication ne serait rien d'autre en fin de compte que de «faire penser à des publics cibles ce qu'on veut qu'ils pensent afin qu'ils fassent ce qu'on veut qu'ils fassent » (Schwebig, 1988). L'individu est une cible à atteindre, une ressource à activer pour assurer la performance de l'entreprise. La situation du communicant est inconfortable : diffuser une information passée au tamis des éléments de langage et constater jour après jour, au sein du corps social, le peu de crédit de la parole officielle ou de la belle image projetée. D'une certaine façon, l'action du communicant dans ces conditions ne fait bien souvent que renforcer les tensions et les contradictions de l'entreprise.

Or, dans cette période de crise et de mutation, l'enjeu premier notamment pour les salariés est sans doute moins l'adhésion - au demeurant toujours improbable - que la compréhension. Il importe pour des raisons de sens et de cohésion de comprendre où l'on va: «Plus ça se transforme, plus la compréhension du sens, des contraintes et des finalités devient essentielle ${ }^{87}$. À vrai dire, les tensions de l'entreprise font advenir une nouvelle donne qui met en question et conduit à revisiter les formes mêmes de la communication ${ }^{88}$. Elle oblige à prendre langue avec l'autre, à débattre, à communiquer au vrai sens du terme, c'est-à-dire à échanger et à mettre en commun des arguments contradictoires. La compréhension ne naît pas de l'imposition. Les communicants ont, dans cette perspective, un rôle primordial à assurer : celui de passeurs de sens et de médiateurs. Par l'organisation de dialogues et de confrontations, ils doivent permettre à l'entreprise d'éviter ce grand écart entre l'image projetée et la réalité, écart qui favorise le désengagement et la défiance des salariés.

Le problème est que ce rôle n'est encore que rarement attendu de la part des communicants. Pourtant, des frémissements sont perceptibles, qui témoignent d'un questionnement de ces professionnels et de possibles reconfigurations de l'approche de la communication dans les entreprises, loin des seules approches instrumentales ou indexées sur le seul pouvoir de la marque. De nouvelles formes de communication plus interactives (réseaux sociaux par exemple) émergent contribuant à déplacer les lignes habituelles d'une com' sous contrôle. Les communicants sont, de fait, confrontés à de forts enjeux de métier et d'identité

\footnotetext{
${ }^{87}$ Ce verbatim sans indication d'auteur est tiré des entretiens que nous avons menés avec des communicants. Il en sera de même pour les suivants.

${ }^{88} C f$. Dominique Wolton, " Pourquoi la communication des entreprises est devenue intenable ", Le Monde, 19 novembre 2012.
} 
professionnelle ${ }^{89}$. L'accroissement des tensions, des aléas, des problèmes, l'ouverture à de nouvelles pratiques de dialogue font croître le besoin de professionnalisme, mais dans une acception plus étendue que celle qui a cours. Faire face à des imprévus, des situations difficiles, des crises, organiser des débats, des échanges demande des qualités autres que celle d'un exécutant de plan de com'. Une «dynamique de métier » (Osty, 2002) est à l'œuvre chez ces professionnels à travers les situations variées qu'ils rencontrent, un maillage de relations multiples et d'épreuves diverses à la fois stratégiques et quotidiennes.

On peut le constater notamment à travers des actions développées par l'Association française de communication interne (AFCI). Nous nous appuierons ici sur deux d'entre elles - une formation et un groupe d'analyse de pratiques - qui, chacune à sa façon, traduit une volonté de comprendre pour agir autrement. Sans doute faut-il rappeler qu'il s'agit là de démarches menées dans le cadre d'une association et qu'elles ne concernent pas toute la communauté des communicants. Démarches émergentes, certainement limitées, mais qui n'en sont pas moins significatives d'ouverture en cours dans ce champ professionnel.

\section{La formation : des professionnels en quête de savoirs}

De janvier à avril 2014, l'AFCI a organisé la cinquième édition d'un cycle court de formation intitulé Bien communiquer aujourd'hui : l'apport indispensable des sciences sociales. Cette formation s'inscrit dans une réflexion plus large engagée par l'association, début 2009, sur les savoirs nécessaires aux pratiques professionnelles des communicants. Son objectif: proposer aux professionnels, déjà détenteurs de compétences, des éléments de connaissances afin de penser la communication et d'agir dans une période où le rôle de l'entreprise dans la société est fortement interrogé. Cette initiative ${ }^{90}$ témoigne du souci de l'AFCI de mettre à distance une communication faisant la part belle à l'image et aux outils au profit d'une communication plus sociale et au cœur des situations de travail et des relations entre les individus.

\footnotetext{
${ }^{89}$ Sur ces deux sujets, on peut revenir avec bonheur sur les travaux de Florence Osty (2002) et sur ceux, plus anciens, de Renaud Sainsaulieu : L'Identité au travail (1977 mais nouvellement réédité avec une préface de Norbert Alter 2014).

${ }^{90}$ Dans la continuité de celle-ci, d'autres ont eu lieu (conférence-débat intitulée Changer de regard: quand les sciences sociales et les entreprises se rencontrent en juin 2012) ou sont en cours : restitution d'une seconde série d'entretiens (juin 2013), rencontre à venir afin de créer des collaborations entre enseignants-chercheurs et praticiens (18 juin 2013), conférence-débat intitulée Parole au travail, parole sur le travail (4 juillet 2013).
} 
Tout est parti d'un séminaire organisé en mars 2007 sur l'apport des sciences humaines et sociales (SHS) à la communication. Ce séminaire a été suivi d'une interpellation propre à l'association, rappelant le rôle premier de la communication, à savoir le rapport à l'autre bien avant tous les plans, outils ou autres dispositifs : "Quels sont nos fondamentaux scientifiques, et surtout comment assurons-nous le lien permanent entre le matériel analytique et conceptuel qu'ils nous proposent et les pratiques que nous développons? »(Besse, 2008). Cette question et sans doute aussi le contexte qui est venu accélérer le questionnement des praticiens sur leur rôle ont suffisamment porté pour qu'un groupe de travail, réunissant communicants, consultants et chercheurs, soit constitué en janvier 2009. Dès le départ, la vocation de cet atelier a été à la fois de développer des réflexions et d'en trouver une traduction à travers des actions ou des événements. Une enquête par entretien ${ }^{91}$ a été initiée afin de comprendre la place et le rôle de la communication dans l'entreprise, son évolution et d'analyser la nature du rapport des praticiens avec les SHS dans leur activité. Parmi les constats tirés, celui de la nécessité d'un socle de savoirs en plus des savoir-faire de métier.

À leur manière, les professionnels appellent à un nouvel équilibre entre compétences et capacités analytiques, jusqu'alors sous-estimées, mais seules susceptibles de capter des éléments de connaissance du corps social, de les analyser et de les utiliser pour agir. Or, ces capacités d'analyse puisent à la source des SHS, dont l'utilisation apparaît comme un enjeu pour les communicants ${ }^{92}$. Certes, tous ne s'y réfèrent pas spontanément ni explicitement. Certains évoquent quelque chose qui relève de la connaissance. D'autres en parlent plus explicitement : "J'ai découvert que les SHS étaient des clés de compréhension de phénomènes qu'on ne peut pas comprendre autrement $»$. La majorité comprend qu'elles proposent des clés de compréhension importantes pour dénouer les complexités de l'entreprise et agir sur ses dynamiques communicationnelles.

Rédigé en décembre 2009, le communiqué de presse annonçant la mise en place de la formation sur l'apport de ces sciences aux pratiques de communication, reprend les principaux éléments de ce constat. L'association y remarquait alors que les professionnels «ont besoin de renforcer les liens entre savoirs fondamentaux et pratiques afin d'accompagner les projets des entreprises et de (re)créer du lien social». Les SHS peuvent aider «à comprendre les situations et les acteurs, à

\footnotetext{
${ }^{91}$ Une première série d'entretiens a été conduite d'octobre 2009 à mars 2010. Une restitution a été organisée au siège d'AXA en juillet 2010, réunissant professionnels et universitaires. Une seconde série d'entretiens a pris fin en avril 2013 et a été restituée le 18 juin 2013.

${ }^{92}$ L'utilisation des apports des SHS (20\% des réponses) apparaît pour la première fois comme une évolution souhaitable de la communication interne dans le baromètre de la fonction communication interne de 2009 réalisé par le cabinet Inergie pour le compte de l'AFCI [en ligne http://www.afci.asso.fr/publication/barometre-inergie-afci-2012-de-la-fonction-communicationinterne].
} 
interroger les pratiques et à agir de façon plus pertinente». La formation est présentée comme une possibilité de "réactiver des connaissances utiles pour mieux comprendre les situations de transformation actuelles, de se confronter à d'autres modes de pensée et d'interrogations, permettant de trouver des repères pour l'action, de participer à des échanges constructifs entre professionnels ». En trois jours, à raison d'une journée toutes les trois semaines, l'association propose depuis 2010 à ses adhérents des éclairages théoriques sur les transformations des organisations et leur contexte social. Parmi les SHS convoquées dans le cadre de cette formation : la sociologie, la sémiologie, les sciences de l'information et de la communication (SIC), les sciences de gestion, la psychosociologie, et l'ergonomie ${ }^{93}$. La première journée propose aux communicants de «comprendre les transformations actuelles de l'entreprise et les relations sociales » avec l'aide de la sociologie et de l'ergonomie. La seconde journée permet de «situer la communication au cour du travail et des processus de changement» avec les apports des SIC notamment. La troisième d'«identifier la place de l'individu au travail et les enjeux du management dans la communication de proximité » grâce aux éclairages de la psychosociologie et des sciences de gestion.

Chaque séance est animée conjointement par un représentant de l'AFCI - JeanMarie Charpentier - et de l'APSE - Jacques Viers. Invariablement, elle débute par un rapport d'étonnement subjectif de «ce qui a été appris» lors de la séance précédente et par les commentaires des participants sur cette même séance. Elle se poursuit par l'intervention du chercheur invité et par les remarques d'un grand témoin, directeur de la communication, des ressources humaines ou cadre dirigeant d'une entreprise, permettant de mettre en perspective l'intervention du chercheur invité. Elle se termine enfin par un échange avec les participants, toujours riche du fait de leur intérêt et de la taille des promotions (une vingtaine de participants ${ }^{94}$ ).

S'agissant en particulier des SIC, une discipline paradoxalement peu connue, pour ne pas dire inconnue des praticiens alors même qu'ils exercent en communication, le parti pris a été de montrer en quoi la communication est plus que jamais au cœur du fonctionnement des entreprises comme du travail. Moins le travail est prescrit, plus il est nécessaire de dialoguer, pour coopérer comme pour régler des conflits. Toute entreprise ne peut alors se maintenir et fonctionner qu'en s'incarnant dans les actions et les relations des individus qui la composent, dans les outils qu'ils utilisent, dans les textes qu'ils produisent, dans les conversations qu'ils échangent. La

\footnotetext{
${ }^{93}$ Parmi les intervenants de cette formation, citons notamment Norbert Alter, Vincent Brulois, Mathieu Detchessahar, Florence Giust-Desprairies, François Hubault, Florence Osty, Maurice Thévenet, Marc Uhalde, Philippe Zarifian...

94 À ce jour, une centaine de praticiens ont suivi ce cycle de formation depuis 2010 ; pour la plupart responsables de communication interne, quelques directeurs de la communication, ainsi que quelques consultants. En règle générale, ce sont des praticiens expérimentés et confirmés.
} 
communication est donc en première ligne car les «modes de relation» et les «manières de parler » au travail se transforment (Giroux et Giordano, 1998).

\section{Le groupe d'analyse de pratiques: une amorce de démarche réflexive}

Parallèlement, un groupe d'analyse de pratiques (GAP) a été mis en place pour les communicants ayant participé à la formation. Dès 2011 en fait, en même temps que démarrait la deuxième saison de la formation. De façon concrète, cette initiative est née d'un double constat. D'une part, les participants eux-mêmes souhaitaient donner une suite à la formation après avoir vécu avec une certaine intensité les trois journées. L'expérience leur laissait un goût de trop peu et surtout leur faisait entrevoir de nouvelles façons d'envisager leur activité quotidienne à partir des SHS. En clair, ils demandaient d' «aller plus loin ». D'autre part, deux des chercheurs au moins, intervenant dans le dispositif de formation - en l'occurrence la sociologue Florence Osty et la psychosociologue Florence Giust-Desprairies -, avaient été frappées par la nature des questionnements de communicants aux prises avec des réalités professionnelles et personnelles parfois délicates, entre tensions et dilemmes, entre action et prise de distance.

Ce double constat a débouché, après réflexions méthodologiques avec les deux chercheurs, sur la construction d'une offre faite aux sessionnaires de participer à une nouvelle expérience sous la forme d'un groupe d'analyse de pratiques. À la différence de la formation, conçue spécifiquement dans le cadre de l'AFCI, le dispositif des GAP existe par ailleurs et se développe dans plusieurs champs professionnels : l'enseignement, le secteur médico-social, le conseil, etc. ${ }^{95}$ Il a paru aux initiateurs de la formation et aux deux chercheurs que le dispositif pouvait correspondre aux attentes et réalités professionnelles des communicants. Alors que la formation s'appuie sur différents apports disciplinaires en sciences sociales, le GAP part des situations et problèmes des professionnels pour les soumettre à l'analyse collective avec l'appui d'un chercheur, le plus souvent sociologue ou psychosociologue.

Le premier GAP a été lancé en juin 2011 et s'est déroulé avec une douzaine de participants, formés au cycle court de l'AFCI en 2010, sur quatre séances d'une demi-journée. Un second a été mis en place en septembre 2012 et un troisième commence à l'automne 2013. Concrètement, une séance se déroule de la façon suivante. Le groupe procède à un tour de table, chacun évoquant brièvement une situation, un cas, un problème. Les participants se mettent d'accord sur le cas qu'ils vont investiguer ensemble. Le praticien dont le cas est retenu fait alors un récit plus

\footnotetext{
${ }^{95}$ À ce propos, on lira avec intérêt le n ${ }^{\circ} 11$ de la Nouvelle revue de psychosociologie paru en 2011.
} 
détaillé de la situation à laquelle il a été confronté, et cela sans grille préétablie, en restant au plus près d'un récit subjectif. Les participants posent ensuite des questions d'éclaircissement sur le récit. Chacun fait part de ce qu'il comprend de la situation, sans jugements, sans préconisations. C'est à proprement parler un temps d'analyse collective dans une logique compréhensive. Le chercheur intervient enfin pour identifier, à partir des traits dominants dégagés par le groupe, des éléments de connaissance, notamment en termes d'identité professionnelle et de métier.

Quelques principes de fonctionnement doivent être retenus : un engagement de participation à toutes les séances, la confidentialité qui donne la possibilité d'une parole sans risque pour les individus, un climat de bienveillance dans le groupe à la fois pour soi et pour les autres, la possibilité de produire pour l'usage exclusif du groupe une trace sous la forme d'un compte-rendu des séances. Cette méthode permet de dégager du sens pour tous et de déconstruire collectivement une complexité vécue et portée individuellement. Elle offre l'occasion de mettre au jour des dimensions propres à une culture de métier. Elle a permis en l'occurrence aux communicants de prendre du recul par rapport à leur quotidien et ainsi d'amorcer une démarche de type réflexif permettant à la fois prise de conscience et examen approfondi du vécu professionnel. Il faut dire qu'un tel groupe ne peut véritablement fonctionner sans un investissement fort des participants. L'expérience a montré que de tels groupes offrent l'occasion d'aller assez loin sur le plan professionnel et personnel, d'articuler de façon originale individuel et collectif.

Une des participantes a rapporté dans un article ${ }^{96}$ paru dans la revue de l'AFCI son expérience du premier GAP sans pour autant enfreindre la règle de confidentialité propre au groupe. Cette écriture de l'oralité des séances s'est même faite avec l'accord unanime des autres participants de la session. Elle a retenu plusieurs dimensions se rapportant aux communicants : un fort besoin de s'exprimer sur leur ressenti plutôt que sur les pratiques professionnelles indiquant la forte charge émotionnelle liée à la fonction; une propension à aller directement aux préconisations voire aux solutions tant le communicant est attendu sur son opérationnalité plutôt que sur son diagnostic ou son analyse ; une difficulté parfois à porter la légitimité du métier et son identité professionnelle... Autant de dimensions soutenant l'intérêt de tels groupes pour identifier ce qui se joue dans les organisations et renforcer la place du communicant «exposé en première ligne, en tant que témoin, acteur et sujet» (Blanc-Michelland, 2012).

Alors que la formation s'appuie d'abord sur la présentation d'apports théoriques en SHS possiblement utiles aux praticiens, le principe d'un GAP est de partir des situations et des pratiques professionnelles des communicants pour les soumettre à une analyse collective encadrée. Il s'agit de pousser plus loin la mise à distance des

${ }^{96}$ (Blanc-Michelland, 2012). 
pratiques professionnelles, moins par l'apport d'un outillage conceptuel que par la mise en place d'un dispositif permettant la déconstruction de situations de travail et une réflexion a posteriori. L'objectif est de les encourager à mettre en mots des situations vécues et douloureuses, de les aider à comprendre les logiques d'action à l'œuvre pour mettre ces situations à distance. En fin de compte, par l'intermédiaire de ce travail partagé sur soi, se dessine progressivement une autre posture professionnelle. Cela représente tout à la fois un faible gain et une grande avancée tant le communicant interne "n'a pas de métier clairement défini et souffre, de ce fait, d'un déficit de légitimité» (idem). Bien plus que la formation, le GAP leur demande ainsi un engagement fort - presque intime - non seulement à être présent à l'ensemble des séances, mais aussi à une participation active dans le groupe qui rompt avec « une certaine forme de solitude » à laquelle le prédispose son activité (idem).

\section{Décoder pour repenser les pratiques professionnelles}

Convenons que cet engouement pour les SHS n'a rien d'évident. Pendant longtemps, un parti pris désignait les salariés comme des individus «qui étaient surtout agis, le plus fréquemment à leur insu, par des déterminants sociaux» (de Singly, 2002). Leurs comportements étaient vus comme socialement déterminés sans que les individus eux-mêmes n'en aient conscience. Mais les complexités nouvelles auxquelles sont soumises les entreprises changent la donne. La doxa managériale a du mal à se renouveler et n'aide pas, c'est le moins qu'on puisse dire, à lire et à comprendre les transformations de l'entreprise moderne dans un contexte mondialisé, croisant les cultures, les modes d'action et les intérêts. Le territoire de l'action s'agrandit, mondialisation aidant, pendant que le temps de l'action se réduit sous le coup du développement des technologies d'information et de communication. L'action se confond bien souvent avec une simple réaction à des événements qui échappent pour partie aux directions d'entreprise. En définitive, les incertitudes augmentent et, avec elles, les risques. Le référentiel managérial est pour une large part «sans boussole » (Segrestin, 2007). Les individus ne s'y retrouvent plus. Le « reporting a remplacé le raisonnement» (Tixier, 2010), déresponsabilisant les décideurs, plaçant les managers dans un «entre-deux managérial» (Flamant, 2002) et sur-organisant le travail des salariés. Tout nouvel outil de gestion est perçu comme une fuite en avant, plutôt que porteur de sens, et renforce la quête de repères des individus.

Dans ce contexte, les SHS se présentent comme un moyen pour des professionnels de comprendre, de décoder, de trouver du sens par eux-mêmes. La perte de repères pousse de façon générale l'individu à être réflexif et à interpréter. Anthony Giddens (1994) y voit les symptômes d'une transformation des normes et des modes 
d'action, conséquences de la modernisation de nos sociétés. Il fait une place à part à la réflexivité, c'est-à-dire à cette capacité d'un individu à examiner en permanence ses actions, dans une sorte de retour sur ses pratiques. Lorsque les modes d'action habituels - les routines - ne sont plus efficaces ou pertinents, l'individu est conduit à réfléchir à un autre mode d'action à partir de la situation dans laquelle il se trouve. Le savoir devient un élément de la révision de ses pratiques sociales. Selon Giddens, ce processus d'« examen et [de] révision constante des pratiques sociales, à la lumière des informations nouvelles concernant ces pratiques ", c'est-à-dire les connaissances acquises, caractérise les sociétés modernes, en tout cas «fait intimement partie du tissu des relations modernes » (idem). L'individu n'est plus un simple agent n'ayant pas conscience de ce qu'il fait, mais un acteur qui s'interroge ; car la réflexion précède l'action. C'est pourquoi, conclut-il, «la modernité est profondément et intrinsèquement sociologique» (idem), pointant, parmi les SHS, la place singulière occupée, selon lui, par la sociologie.

Le travail de réflexivité, dans le cas qui nous occupe, est poussé par la volonté du communicant d'interpréter ce qui l'entoure et ce qui entoure son action. Réflexivité et interprétation se complètent. Elles supposent d'une manière ou d'une autre la nécessité de l'arrêt, pour un temps, de l'action en continu que l'on fait sans réfléchir ou presque - toujours la routine - et de mise à distance de cette action pour lui donner du sens et, potentiellement, une autre forme. Selon les termes d'Yves Citton, interpréter c'est faire un "effort de compréhension ", un "effort visant à donner sens à un ensemble d'indices, traces, signes, venus d'ailleurs et d'autrui ", une "certaine façon de rendre significatifs des éléments d'information disparates »" accumulés au fil de l'action, sans s'y arrêter (2010). Mise à distance, compréhension, sens, nous retrouvons là trois des constats que nous avions établis à partir d'une première série d'entretiens auprès de communicants ${ }^{98}$. Immergés sans recul dans la société de communication, ils ont pu avoir la tentation de croire, comme d'autres, que l'important est que l'information, toute l'information, circule, circule vite. Mais, du fait de leur fonction, ils comprennent mieux que d'autres qu'une telle communication informe peu et ne fait pas lien - quatrième de nos constats. Informe peu car la quantité ne fait pas la qualité. La difficulté n'est plus de trouver de l'information, mais de construire une information intéressante et qui fasse

\footnotetext{
${ }^{97}$ L'auteur précise que cet effort relève « autant d'un esprit de finesse que d'un esprit de géométrie ». En effet, il «tente, d'une part, de rester aussi proche que possible de ce que l'ensemble de signes analysés paraît dire par lui-même depuis toujours à quiconque veut bien l'entendre, mais [...] il tente simultanément et d'autre part, d'apporter des effets de compréhension qui recomposent ces signes de façon à y faire reconnaître un sens inédit » (2010).

${ }^{98}$ Cf. Vincent Brulois et Jean-Marie Charpentier, « De l'image au social : le chemin d'une évolution pour les métiers de la communication », $79^{\text {e }}$ Congrès de l'ACFAS, Colloque Perspectives de développement des pratiques de communication: modes d'organisation et enjeux de formation, Université de Sherbrooke, 11 et 12 mai 2011.
} 
sens. Ne fait pas lien car le trop plein d'informations détourne les individus d'une telle communication. En bref, informer n'est pas communiquer ${ }^{99}$.

Dans les propos des communicants revient plusieurs fois la volonté de ralentir le temps, voire de l'arrêter, ne serait-ce qu'un instant, afin de sortir du flux incessant d'informations engendré par l'accélération provoquée par les réseaux numériques. Hartmut Rosa (2010) encourageait, il y a peu, les individus à se créer des « mécanismes de retard et de ralentissement » afin qu'une action n'en entraîne pas automatiquement une autre, prônant l'aménagement d' "oasis de décélération » afin de ralentir le rythme quotidien des activités dans lesquelles nous sommes pris. En son temps, Gilles Deleuze (1990) proposait de «ménager des vacuoles de solitude et de silence à partir desquelles [les gens] auraient enfin quelque chose à dire ». Un nouveau rapport au temps apparaît alors comme une condition sine qua non. Pour le communicant, c'est, entre autres, la possibilité de «se donner les conditions nécessaires à avoir quelque chose d'autre à dire que ce qui répond aux questions qui nous sont posées quotidiennement» (Citton, 2010), c'est oser user d'une capacité de résistance ou d'une capacité de critiquer, voire de contredire ${ }^{\mathbf{1 0 0}}$ sa propre entreprise. L'exercice est difficile mais salutaire. Salutaire notamment pour l'entreprise, car «c'est le fait même d'être confronté à la critique qui donne vie aux institutions » remarque Luc Boltanski (2012). Un exercice d'autant plus important que nous savons depuis longtemps qu'aucun individu ne peut agir en suivant étroitement et uniquement des règles édictées par d'autres. On ne peut l'empêcher de réfléchir et d'interpréter. La critique, «en paroles et surtout en actes », rend nécessaire la réflexivité et met en œuvre l'interprétation (idem). "Ce que les institutions édictent n'est justifiable qu'à la condition de demeurer ouvert à la critique et à l'interprétation ", ajoute le sociologue (idem).

Entre temps court et temps long, la difficulté reste bien sûr, pour un professionnel, de conjuguer trois rythmes : celui de l'action, celui de l'adaptation aux changements, celui de la réflexion, de l'analyse, de la mise à distance. Rythmer, c'est désigner ce qui est continuité ou rupture dans le mouvement permanent des changements. La «capacité à décoder» les collectifs ancrés dans une histoire et une culture, les communautés qui se font et se défont au gré des projets, les environnements qui se décloisonnent et se recoupent, apparaît, aux yeux d'un nombre croissant de communicants, comme une clé qui les fera sortir du monde des apparences et de la belle image. S'impose aussi l'idée qu'un décodage par les SHS peut favoriser la compréhension du contexte, des enjeux et, potentiellement, nourrir d'autres pratiques professionnelles.

\footnotetext{
${ }^{99}$ Selon le titre de l'ouvrage de Dominique Wolton (CNRS éditions, 2009).

${ }^{100}$ Selon le titre de l'ouvrage collectif - Contredire l'entreprise - dirigé par Thierry Libaert, Andréa Catellani et Jean-Marie Pierlot (Presses Universitaires de Louvain, 2010).
} 
L'appel à communication de ce colloque nous invitait à « réinterroger le socle des compétences communes et des compétences spécialisées nécessaires à l'exercice des métiers de la communication», à "saisir l'occasion», nous chercheurs ou praticiens, «de repenser les pratiques professionnelles en communication». À l'aune de ce que nous venons de relater, le moment paraît favorable. Soif d'apprendre et de connaitre des communicants, de discuter avec des chercheurs, envie de répondre à une forme de demande sociale ${ }^{\mathbf{1 0 1}}$ de la part de certains chercheurs, renforçant le rôle des uns et des autres. D'une certaine façon, et c'est heureux, il n'y a plus «d'acteurs sociologiquement naïfs 》 (Smelser, 1998 dans Wallerstein, 1998). Ce changement engage de plus en plus de professionnels sur le terrain de la réflexivité. Pour la plupart, ils sont plus informés, mais aussi plus sceptiques et plus critiques. En entreprise, cela oblige alors tout le monde, des dirigeants aux salariés, à penser différemment et à modifier les relations de travail.

Trois éléments, nous semble-t-il, permettent d'apprécier la «dynamique de métier» (Osty, 2002) en cours chez un certain nombre de communicants. Il y a d'abord ce qui se passe du côté des savoirs propres au métier. Le fait de chercher à dépasser les seuls savoir-faire peut être vu comme un facteur de maturation professionnelle. Dans leur contexte de travail, les communicants sont d'abord attendus sur leur opérationnalité - afin d'agir. Mais les évolutions de ce contexte, comme de l'entreprise toute entière, les poussent à rechercher des savoirs plus théoriques, des «fondamentaux scientifiques» disent-ils. Sans forcément être en capacité de préciser leur attente, certains ressentent pourtant le besoin de méthodes de réflexion - afin de comprendre : "Il faut être à la fois dans l'opérationnel et dans la réflexion. Il faut avoir une ouverture d'esprit pour comprendre les choses, [...] pour comprendre les acteurs, leur stratégie, la façon dont ils se positionnent par rapport à un changement ou un projet ». Il y a ensuite ce qui se passe du côté de l'identité de métier. À certains égards, on retrouve là des professionnels plus solides qu'il y a quelques années, plus exigeants aussi. Des professionnels qui cherchent à renforcer leur construction identitaire à travers l'expérience, souvent difficile, de leur travail et à travers l'utilité sociale de leur métier. Certes, il n'y a pas d'identité professionnelle uniforme chez les communicants ; mais la tendance au renforcement de celle-ci est manifeste, notamment chez ceux qui travaillent en communication interne. Il y a enfin ce qui se passe du côté de la régulation de métier. C'est un métier qui construit ses règles aux interfaces. Le communicant est en relation directe avec quantité d'acteurs (managers, opérationnels, RH, salariés) sur plusieurs scènes.

\footnotetext{
${ }^{101}$ Nous reprenons à notre compte la définition qu'en a fait Robert Castel. La demande sociale est «le système d'attentes de la société à l'égard des problèmes quotidiens qui la sollicitent aujourd'hui »; elle est plus ou moins spontanée, masquée, confuse (« La sociologie et la réponse à la demande sociale » dans B.Lahire (dir.), À quoi sert la sociologie?, La Découverte, 2002.
} 
D'où des modalités d'action et de régulation diverses révélant du même coup force et fragilité du métier.

Il y a toujours une certaine intériorisation, voire un complexe lié à une certaine représentation dévaluée de la com'. Mais, par ses multiples relations, son réseau d'interactions, le communicant interne se connecte de plus en plus avec le système social. Là se joue, sans doute, des choses importantes pour lui. Son champ d'intervention au fond, c'est le social au sens large dans l'entreprise. Il n'est pas seul dans ce cas, mais ça lui confère une responsabilité particulière à la dimension éthique (éthique du métier, éthique professionnelle) forte. Elle renvoie aussi à une certaine conception de l'entreprise, du lien, des relations entre les acteurs, de la place des salariés. Une certaine façon d'être ensemble, de faire société en entreprise.

Dès lors, on comprend mieux pourquoi le communicant a besoin de savoirs nouveaux pour circuler entre les mondes sociaux ${ }^{\mathbf{1 0 2}}$ présents dans l'entreprise. Du fait de son activité, il est confronté plus qu'un autre aux diverses manifestations du social qui appellent un développement du dialogue avec et entre les salariés: développement de dialogues critiques ${ }^{103}$, d'espaces de discussion ${ }^{104}$. Il ne fait plus de doute aujourd'hui que la communication est au cœur du lien social, qu'elle est un « activateur du changement social» (Miège, 1989). Au fond, la communication est d'abord une question sociale, alors qu'on la cantonne le plus souvent et depuis longtemps aux seules dimensions d'image, de marque et de message. Évolution rassurante selon nous, ce constat paraît partagé aujourd'hui par de plus en plus de communicants.

\section{Bibliographie}

Alter, N. (2000). L'Innovation ordinaire, Paris, France : éditions PUF.

Basilien, J-P., Gilabert, M. et Monneuse, D. (2010, octobre). Découplages : le social sous contrainte, Note de conjoncture sociale d'Entreprise \& Personnel, 292.

Besse, J-M. (2008, avril). Les sciences humaines n'intéressent-elles plus les communicants ? Les Cahiers de la communication interne, 22, 1.

\footnotetext{
${ }^{102}$ Selon le titre de l'ouvrage de Florence Osty, Renaud Sainsaulieu et Marc Uhalde : Les Mondes sociaux de l'entreprise (La Découverte, 2007).

${ }^{103}$ Cf. Armand Hatchuel, «Dialoguer et critiquer, un travail nécessaire », Le Monde, 12 janvier 2010.

${ }^{104}$ Cf. Matthieu Detchessahar, Les Déterminants organisationnels et managériaux de la santé au travail, synthèse de l'étude SORG pour l'Agence nationale de la recherche, septembre 2009 [en ligne http://www.pedagogie.acnantes.fr/servlet/com.univ.collaboratif.utils.LectureFichiergw?CODE FICHIER=1282858626760 \&ID FICHE $=1282682070788$.
} 
Blanc-Michelland, C. (2012, décembre). Paroles de communicants. Les Cahiers de la communication interne, 31, 20-22.

Boltanski, L. (2012, 12 juillet). Notre avenir est-il démocratique ? Le Monde.

Callon, M., Lascoumes, P. et Barthe, Y. (2001). Agir dans un monde incertain, Paris, France : éditions du Seuil.

Citton, Y. (2010). L'Avenir des humanités, Paris, France : éditions La Découverte.

Deleuze, G. (1990). Pourparlers, Paris, France : éditions de Minuit.

Flamant, N. (2002). Une Anthropologie des managers, Paris, France : éditons PUF.

Giddens, A. (1994). Les Conséquences de la modernité, Paris, France : éditions L'Harmattan.

Giroux, N. et Giordano, Y. (1998, septembre-octobre). Les deux conceptions de la communication du changement. Revue française de gestion.

Miège, B. (1989). La Société conquise par la communication, Grenoble, France: éditions PUG.

Osty, F. (2002). Le Désir de métier, Rennes, France : éditions PUR.

Rosa, H. (2010). Accélération. Une critique sociale du temps, Paris, France: éditions La Découverte.

Schwebig, P. (1988). Les Communications de l'entreprise, Paris, France : éditions Mc Graw-Hill.

Segrestin, D. (2007). Pour une sociopolitique du management. Dans J-C. Sardas, D. Giauque, A-M. Guénette (dir.), Comprendre et organiser. Quels apports des sciences humaines et sociales?, Paris, France : éditions L'Harmattan.

Segrestin, B. et Hatchuel, A. (2012). Refonder l'entreprise, Paris, France : éditions du Seuil.

de Singly, F. (2002). La sociologie, forme particulière de conscience. Dans B. Lahire (dir.), À quoi sert la sociologie?, Paris, France : éditions La Découverte.

Tixier, P-É. (dir.) (2010). Ressources humaines pour sortie de crise, Paris, France : Sciences Po Les Presses.

Wallerstein, I. (1998). L'héritage de la sociologie, la promesse de la science sociale. Les Cahiers de recherche sociologique, 31. 